\title{
Regulation of rat MOR-1 gene expression after chronic intracerebroventricular administration of morphine
}

\author{
ZHI-PING ZHU, RAMESH B. BADISA, DONALD E. PALM and CARL B. GOODMAN \\ College of Pharmacy and Pharmaceutical Sciences, Florida A and M University, Tallahassee, FL 32307, USA
}

Received July 17, 2011; Accepted October 19, 2011

DOI: $10.3892 / \mathrm{mmr} .2011 .677$

\begin{abstract}
The $\mu$-opioid receptor is the primary site for the action of morphine. In the present study, we investigated the regulation of the $\mu$-opioid receptor mRNA levels in the locus ceruleus, ventral tegmental area, nucleus accumbens and hypothalamus of the rat brain following intracerebroventricular administration of morphine for 7 days. The isolated mRNA from these regions was subjected to real-time quantitative RT-PCR to determine the regulation of $\mu$-opioid receptor gene expression. It was observed that 7 days of treatment with morphine significantly down-regulated the $\mu$-opioid receptor mRNA levels in the hypothalamus of the brain in comparison to the control group. However, the $\mu$-opioid receptor levels in the locus ceruleus, ventral tegmental area and nucleus accumbens regions remained the same as the control levels. Down-regulation of $\mu$-opioid receptor mRNA levels in the hypothalamus region of the brain indicates the probable role of opioids to influence neuroendocrine function. The results further indicate that cellular adaptation for morphine tolerance is tissue-specific. These findings help us to understand the mechanism of morphine tolerance in various regions of the brain.
\end{abstract}

\section{Introduction}

Opiate analgesics are widely used and abused drugs throughout the world. These drugs are shown to alter gene transcription in the brain and spinal cord following acute and chronic administration. Altered gene expression due to opiate exposure is responsible for the behavioral changes in

Correspondence to: Dr Carl B. Goodman, College of Pharmacy and Pharmaceutical Sciences, Florida $\mathrm{A}$ and $\mathrm{M}$ University, Tallahassee, FL 32307, USA

E-mail: carl.goodman@famu.edu

Abbreviations: AOP, anti-opiate peptides; GAPDH, glyceraldehyde 3-phosphate dehydrogenase; ICV, intracerebroventricular; LC, locus ceruleus; MOR-1, mu-opioid receptor; NA, nucleus accumbens; NPFF, neuropeptide FF; VTA, ventral tegmental area; WHO, World Health Organization

Key words: $\mu$-opioid receptor, real-time RT-PCR, rat brain, morphine, tolerance drug addicts. Morphine, which is one of the highly abused opiates, is the major metabolite of heroin. Previous studies have demonstrated that morphine induces long-term changes in neurons (1). Although morphine is one of the most abused drugs, it is widely employed to relieve various forms of acute and chronic pain (2). The World Health Organization (WHO) recommended its use for controlling pain in cancer patients (3). However, its repeated administration is shown to result in the development of tolerance, where the response at a given dose decreases and higher doses are required to attain the same original effect (4). The process of morphine tolerance is very complex and involves a number of cellular and multi-cellular adaptations, which are not yetwell understood as yet (5). It was shown that $\mu$-opioid receptor (MOR-1) is the primary site of action for morphine and the other most commonly used opioids (6). From the clinical point of view, it is crucial to understand the mechanism of morphine tolerance, which could lead to treatment and prevention of opiate addiction.

A number of contradictory observations have been reported by various investigators regarding the chronic affect of morphine treatment on MOR-1 regulation in animal models or cultured cells. While a number of groups with animal models showed either down-regulation $(7,8)$ or up-regulation (9), other groups reported evidence of no change in the density of MOR-1 $(10,11)$. The reasons for this discrepancy probably reflect differences in the route of administration, dosage used, animal species, number of days of treatment and the area of the brain region analyzed for regulation. The inconsistency in the regulation of MOR-1 in chronic morphine-treated animal models prompted us to re-evaluate the possible alterations of MOR-1 levels.

Earlier, we reported the down-regulation of $\mu$-opioid binding sites in rats following chronic administration of NPFF and morphine in various regions of the brain by quantitative autoradiography, using radiolabelled $\left[{ }^{3} \mathrm{H}\right]$ DAMGO (12). The present study was designed to determine the role of chronic intracerebroventricular (ICV) morphine on the regulation of MOR-1 gene expression levels in discreet brain regions. The locus ceruleus (LC) area in the brain is related to the analgesic effect of morphine, while the hypothalamus was shown to have high levels of MOR-1 (13). Thus, studying the regulation of MOR-1 in LC, the ventral tegmental area (VTA), nucleus accumbens (NA) and hypothalamus tissue regions of the rat brain with administration of morphine will help us to understand the cellular adaptation of these receptors to tolerance. 
We hope that our independent approach may provide the means to determine the changes in MOR-1 mRNA levels due to morphine treatment in animals.

\section{Materials and methods}

Materials. Morphine sulfate was obtained from Sigma Chemical Company (St. Louis, MO, USA) and dissolved in saline at $40 \mathrm{mg} / \mathrm{ml}$, based on our previous experiments (9). ALZET 2001 osmotic minipumps were purchased from Alza Corporation (Palo Alto, CA, USA). All other routine chemicals and reagents used were of analytical grade.

Animals. Male Sprague-Dawley rats weighing 200-240 g were purchased from Harlan (Indianapolis, IN, USA) and utilized in the experiments. All animals were maintained on a 12-h light-dark cycle in a temperature-controlled environment $\left(\sim 21^{\circ} \mathrm{C}\right)$, and were provided with food and water ad libitum. The animals were allowed to acclimatize to the surroundings prior to the start of the studies. All animal studies were performed according to guidelines set out by the Florida A and M University's Animal Ethics Committee. Animals were divided randomly into two groups, with 6 rats in each group. Prior to surgery, all rats were anesthetized with $3 \%$ halothane and positioned in a Stellar stereotaxic frame (Stoelting, Wood Dale, IL, USA), with the incisor bar set at $3.0 \mathrm{~mm}$ below intra-aural zero, as described previously (12). ALZET 2001 osmotic minipumps, containing $200 \mu \mathrm{l}$ of morphine, were implanted SC into the animals. Each pump was connected to a stainless steel ICV guide cannula via plastic tubing $(10 \mathrm{~cm}$ length). The minipumps perfused the lateral cerebral ventricle of the rats for 7 days (chronic), using the Alzet brain infusion kit. Morphine was infused at a rate of $1.0 \mu \mathrm{l} / \mathrm{h}(40 \mu \mathrm{g} / \mu \mathrm{l})$. The control animals also received surgery and were infused with saline at the same flow rate. At the end of the treatment periods, rats were sacrificed by decapitation and their brains were dissected rapidly for LC, VTA, NA and hypothalamus regions. All samples were placed on dry ice and stored at $-80^{\circ} \mathrm{C}$ until the day of assay.

Isolation of RNA. Various tissue samples of rat brain from the respective groups were each individually homogenized with VirTishear polytron homogenizer (Virtis Company, Inc., Cardiner, NY, USA) in $1 \mathrm{ml}$ TRIzol reagent. Total RNA was extracted with chloroform and isopropanol according to the manufacturer's instructions (Invitrogen, Carlsbad, CA, USA). Following ethanol precipitation, the vacuum-dried RNA was dissolved in $200 \mu 1$ of DEPC-water and the quantity of total RNA was measured by the Nanodrop ND-1000 spectrophotometer (NanoDrop Technologies, Wilmington, DE, USA). RNA was subjected to DNase treatment for $30 \mathrm{~min}$ at $37^{\circ} \mathrm{C}$ using DNase Treatment and Removal Reagent (Ambion, Austin, TX, USA). The integrity of total RNA and the absence of contaminating genomic DNA were confirmed by $1 \%$ agarose gel electrophoresis. The purified RNA with A260/ A280 ratio of $\geq 1.8$ was subsequently used for cDNA synthesis.

cDNA synthesis. The cDNA synthesis was performed with an iScript cDNA synthesis kit (Bio-Rad, Hercules, CA, USA) using $1 \mu \mathrm{g}$ of total RNA according to the manufacturer's instructions.
Real-time quantitative RT-PCR. Real-time quantitative RT-PCR was performed in an iCycler thermal cycler with the MyiQ detection system (Bio-Rad) using iQ Syber Green supermix. MOR-1 and GAPDH products were synthesized by separate PCR reactions, carried out in $50 \mu \mathrm{l}$ final volume with $2 \mu \mathrm{l}$ of cDNA sample and $300 \mathrm{nM}$ of specific primers. The thermal profile was optimized with an initial denaturation of 5 min at $95^{\circ} \mathrm{C}$ (hot start) followed by 40 amplification cycles with a two-step Amp+melt program according to the MyiQ Instructional Manual. The amplification program used was a two-step-protocol: $95^{\circ} \mathrm{C}$ for $10 \mathrm{sec}$ and $55^{\circ} \mathrm{C}$ for $45 \mathrm{sec}$. The specificity of PCR products was checked by the peak of the melt curve or agarose gel electrophoresis when necessary. A control with all reagents with the exception of the cDNA sample was performed in preliminary experiments.

Data analysis. For the sake of normalization of gene expression, GAPDH, a housekeeping gene, was used as the reference gene and amplified in parallel with MOR-1 using real-time PCR. The relative unit of mRNA level (log copy number) was calculated from a fit standard curve using the PCR baseline subtracted $\mathrm{Ct}$ value. The MOR-1 mRNA level in each sample was normalized as a ratio of MOR-1 mRNA/GAPDH mRNA. The changed folds of expressed MOR-1 mRNA level between control and treatment were expressed as a percentage of the control. Significance of the data was analyzed by one-way ANOVA and then compared by Dunnett's multiple comparison tests using GraphPad Prism Software, version 3.00 (San Diego, CA, USA). $\mathrm{P}<0.05$ was considered to be statistically significant.

\section{Results}

Differential regulation of MOR-1 gene expression. The mRNA levels of MOR-1 were quantified by real-time RT-PCR using the MOR-1-specific primers listed in Table I. GAPDH, a housekeeping gene, was used for the normalization of gene expression. It was observed that following 7 days of morphine treatment, the MOR-1 gene expression levels in LC, VTA and NA regions of the rats' brain changed marginally compared to the control (Fig. 1). The percentage of MOR-1 mRNA levels in various brain regions is shown in Table II. The change in the mRNA levels in LC, VTA and NA regions was insignificant $(\mathrm{P}>0.05)$. On the other hand, 7-day morphine treatment caused a significant $(\mathrm{P}<0.05)$ decrease in the MOR-1 mRNA levels in the hypothalamus region of the rat brain. The decrease was found to be $59.4 \%$.

\section{Discussion}

The main aim of this study was to investigate the effect of morphine administration on MOR-1 mRNA levels in the LC, VTA, NA and hypothalamus regions of the rat brain. Even though it is well known that the actions of opiates, such as morphine, are mediated via the MOR-1 (14), the molecular mechanisms governing the MOR-1-mediated transcriptional regulation remain unknown. The mRNA of MOR-1 is very large, ranging between 14 and $16 \mathrm{~Kb}$ (15), but the coding sequence accounts for $<8.5 \%$, while the $3^{\prime}$ un-translated region represents $>90 \%$. The very large size and relatively low abundance of MOR-1 mRNA in the majority of regions 
Table I. Sequence of the primers used in real-time PCR for rat MOR-1 and GADPH.

mRNA accession

(nucleotide position)
Primers

Fragment length

(bp)

\section{Oprm1}

NCBI: NM 013071

5'-CAACTTGTCCCACGTTGATG-3'

(nucleotides 273-391)

5'-TAATGGCTGTGACCATGGAA-3'

GADPH

NCBI: BC 059110

(nucleotides 371-567)

5'-GTCTTCACTACCATGGAGAAGG-3'

GADPH, glyceraldehyde 3-phosphate dehydrogenase.

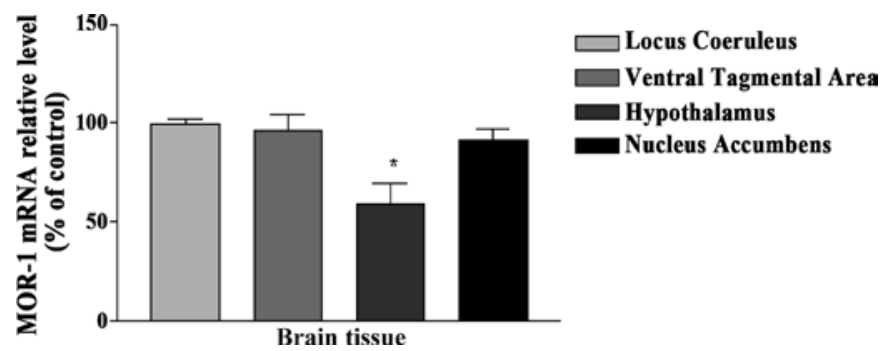

Figure 1. MOR-1 relative mRNA levels were quantified by real-time PCR using GAPDH as the reference gene. The results are the means \pm SEM of a triplicate assay for three independent experiments. "Significance set at $\mathrm{P}<0.05$.

of the central nervous system requires a very highly sensitive method for the measurement of accurate levels of mRNA in tissue extracts. The real-time reverse-transcriptase (RT)-PCR is more specific, sensitive and reproducible in quantifying the initial level of the mRNA in various types of tissues.

In the present study, we utilized the real-time quantitative RT-PCR technique to examine the changes in MOR-1 gene expression in LC, VTA, NA and hypothalamus tissue regions of the rat brain due to exposure of morphine for 7 days by ICV. It was observed that in morphine-treated animals, the MOR-1 mRNA levels decreased significantly $(59.4 \%, \mathrm{P}<0.05)$ in the hypothalamus region, while no changes were detected in LC, VTA and NA regions of the brain in comparison to the control group (Fig. 1, Table II). Similar results were observed in the MOR-1 mRNA levels with the acute morphine treatment (1 day) in LC, VTA and NA regions of the rat brain (data not shown). While the results of our acute morphine treatment were consistent with earlier reports (16), the results of chronic study markedly differed with others (16). One of the reasons for this deviation could be the difference in the number of treatment days; secondly, the route of morphine administration to rats. The present results of the hypothalamus are consistent with earlier reports (15), where decreased levels of MOR-1 mRNA in the hypothalamus upon chronic morphine treatment in rats were shown. The down-regulation of MOR-1 mRNA levels in the hypothalamus indicates that the transcriptional regulation may be one of the mechanisms underlying the reduction of MOR-1 expression.

It was reported that the down-regulation of opioid receptors occurs in two ways. One is due to loss of G-protein coupling, endocytosis and internalization of receptors (17), while the
Table II. Summary of MOR-1 mRNA levels by real-time PCR using GAPDH as the reference gene.

\begin{tabular}{ll}
\hline Locus ceruleus & $99.3 \pm 3.01$ \\
Ventral tegmental area & $96.7 \pm 7.85$ \\
Hypothalamus & $59.4 \pm 10.16^{\mathrm{a}}$ \\
Nucleus accumbens & $91.2 \pm 6.10$
\end{tabular}

The results are the means \pm SEM of triplicate assay for three indepen-

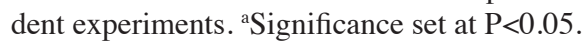

second way involves the ubiquitin/proteasome pathway (18). Splicing during mRNA processing could lead to a number of splice variants and the mRNA, which is devoid of the coding sequence required for the recycling process, would lead to the internalization and down-regulation of the MOR-1 (19,20). It was shown that a splice variant, which was devoid of eight amino acids at the C-terminus sequence in human SK-N-SH cells, could not undergo the recycling process, and thus it led to internalization and down-regulation of the MOR-1 (19). This observation was reconfirmed independently (20).

In the hypothalamus region of the rat brain, it may be possible that ICV infusion of morphine may be localized in this area and that the MOR-1 gene was down-regulated following chronic exposure. On the other hand, in LC, VTA and NA regions of the rat brain, it is possible that MOR-1 may not be fully stimulated with 7-day morphine treatment. In view of this, no down-regulation of MOR-1 gene expression was observed in the LC, VTA and NA regions of the rat brain. Another possibility for unaltered mRNA MOR-1 levels in these regions could be due to interactions of certain endogenous neuropeptides with morphine. This speculation is based on the observation that certain neuropeptides, such as neuropeptide FF (NPFF), are found in high concentration in various regions of the brain (21). NPFF is shown to inhibit a number of morphine-induced effects, such as opposing the development of tolerance and dependence (22). However, further studies are required to prove this assumption.

In conclusion, comparison of mRNA levels in LC, VTA, NA and hypothalamus regions with morphine treatment clearly indicates that the expression of MOR-1 mRNA is highly tissue-specific following chronic ICV morphine treatment in the hypothalamic region. 


\section{Acknowledgements}

This study was supported by the NCRR/RCMI G12 RR03020, the NIGMS/MBRS/SCORE GM08111 and the HRSA SD34HP0 4018 grants.

\section{References}

1. Oliveira MT, Rego AC, Morgadinho MT, Macedo TR and Oliveira CR: Toxic effects of opioid and stimulant drugs on undifferentiated PC12 cells. Ann NY Acad Sci 965: 487-496, 2002.

2. Mao J, Sung B, Ji RR and Lim G: Chronic morphine induces downregulation of spinal glutamate transporters: implications in morphine tolerance and abnormal pain sensitivity. J Neurosci 22 : 8312-8323, 2002.

3. World Health Organization: Cancer Pain Relief. Geneva, 1986.

4. Taylor DA and Fleming WW: Unifying perspectives of the mechanisms underlying the development of tolerance and physical dependence to opioids. J Pharmacol Exp Ther 297: 1-18, 2001.

5. Borgland SL: Acute opioid receptor desensitization and tolerance: is there a link? Clin Exp Pharmacol Physiol 28: 147-154, 2001.

6. Kreek MJ: Opioid receptors: some perspectives from early studies of their role in normal physiology, stress responsivity, and in specific addictive diseases. Neurochem Res 21: 1469-1488, 1996.

7. Bhargava HN and Gulati A: Down-regulation of brain and spinal cord mu-opiate receptors in morphine tolerant-dependent rats. Eur J Pharmacol 190: 305-311, 1990.

8. Tao PL, Lee HY, Chang LR and Loh HH: Decrease in mu-opioid receptor binding capacity in rat brain after chronic PL017 treatment. Brain Res 526: 270-275, 1990.

9. Brady LS, Herkenham M, Long JB and Rothman RB: Chronic morphine increases mu-opiate receptor binding in rat brain: a quantitative autoradiographic study. Brain Res 477: 382-386, 1989.

10. Abdelhamid EE and Takemori AE: Characteristics of mu and delta opioid binding sites in striatal slices of morphine-tolerant and -dependent mice. Eur J Pharmacol 198: 157-163, 1991.

11. De Vries TJ, Tjon Tien Ril GHK, van Der Laan JW, Mulder AH and Schoffelmeer ANM: Chronic exposure to morphine and naltrexone induces changes in catecholaminergic neuro transmission in rat brain without altering mu-opioid receptor sensitivity. Life Sci 52: 1685-1693, 1993.
12. Goodman CB, Emilien B, Becketts K, Cadet JL and Rothman RB: Downregulation of mu-opioid binding sites following chronic administration of neuropeptide FF (NPFF) and morphine. Peptides 17: 389-397, 1996.

13. Sharif NA and Hughes J: Discrete mapping of rat mu and delta opioid receptors using selective peptides: quantitative autoradiography, species differences and comparison with kappa receptors. Peptides 10: 499-522, 1989.

14. Loh HH and Smith AP: Molecular characterization of opioid receptors. Annu Rev Pharmacol Toxicol 30: 123-147, 1990.

15. Brodsky M, Elliott K, Hynansky A, Jenab S and Inturrisi CE: Quantitation of mu opioid receptor (MOR-1) mRNA in selected regions of the rat CNS. NeuroReport 6: 725-729, 1995.

16. Laura JS, Dana ES, Steven ID and Steven RC: Effects of chronic morphine administration on $\mu$ opioid receptor-stimulated $\left[{ }^{35} \mathrm{~S}\right]$ GTP $\gamma$ S autoradiography in rat brain. J Neurosci 16: 2684-2692, 1996.

17. Whistler JL, Chuang HH, Chu P, Jan LY and von Zastrow M: Functional dissociation of mu opioid receptor signaling and endocytosis: implications for the biology of opiate tolerance and addiction. Neuron 23: 737-746, 1999.

18. Chaturvedi K, Christoffers KH, Singh K and Howell RD: Structure and regulation of opioid receptors. Biopolymers 55: 334-346, 2000

19. Bare LA, Mansson E and Yang D: Expression of two variants of the human mu opioid receptor mRNA in SK-N-SH cells and human brain. FEBS Lett 354: 213-216, 1994.

20. Tanowitz $\mathrm{M}$ and von Zastrow $\mathrm{M}$ : A novel endocytic recycling signal that distinguishes the membrane trafficking of naturally occurring opioid receptors. J Biol Chem 278: 45978-45986, 2003

21. Panula P, Aarnisalo AA and Wasowicz K: Neuropeptide FF, a mammalian neuropeptide with multiple functions. Prog Neurobiol 48: 461-487, 1996.

22. Malin DH, Lake JR, Fowler DE, Hammond MV, Brown SL, Leyva JE, Prasco PE and Dougherty TM: FMRF-NH2-like mammalian octapeptide precipitates opiate-withdrawal syndrome in the rat. Peptides 11: 277-280, 1990. 